\title{
Truncating SLC12A6 variants cause different clinical phenotypes in humans and dogs
}

\author{
Mario Van Poucke ${ }^{1} \cdot$ Kimberley Stee $^{2} \cdot$ Laurien Sonck $^{3} \cdot$ Emmelie Stock $^{4} \cdot$ Leslie Bosseler $^{5} \cdot$ Jo Van Dorpe $^{6}$. \\ Filip Van Nieuwerburgh $\mathbb{D}^{7}$. Dieter Deforce $\mathbb{D}^{7}$. Luc J. Peelman ${ }^{1}$. Luc Van Ham ${ }^{2}$. Sofie F. M. Bhatti ${ }^{2}$. \\ Bart J. G. Broeckx $\mathbb{D}^{1}$
}

Received: 28 February 2019 / Revised: 6 May 2019 / Accepted: 14 May 2019 / Published online: 3 June 2019

(c) European Society of Human Genetics 2019

\begin{abstract}
Clinical, pathological, and genetic findings of a primary hereditary ataxia found in a Malinois dog family are described and compared with its human counterpart. Based on the family history and the phenotype/genotype relationships already described in humans and dogs, a causal variant was expected to be found in $K C N J 10$. Rather surprisingly, whole-exome sequencing identified the SLC12A6 NC_006612.3(XM_014109414.2): c.178_181delinsCATCTCACTCAT (p. (Met60Hisfs*14)) truncating variant. This loss-of-function variant perfectly segregated within the affected Malinois family in an autosomal recessive way and was not found in 562 additional reference dogs from 18 different breeds, including Malinois. In humans, SLC12A6 variants cause "agenesis of the corpus callosum with peripheral neuropathy" (ACCPN, alias Andermann syndrome), owing to a dysfunction of this $\mathrm{K}^{+}-\mathrm{Cl}^{-}$cotransporter. However, depending on the variant (including truncating variants), different clinical features are observed within ACCPN. The variant in dogs encodes the shortest isoform described so far and its resultant phenotype is quite different from humans, as no signs of peripheral neuropathy, agenesis of the corpus callosum nor obvious mental retardation have been observed in dogs. On the other hand, progressive spinocerebellar ataxia, which is the most important feature of the canine phenotype, hindlimb paresis, and myokymia-like muscle contractions have not been described in humans with ACCPN so far. As this is the first report of a naturally occurring disease-causing SLC12A6 variant in a non-human species, the canine model will be highly valuable to better understand the complex molecular pathophysiology of SLC12A6-related neurological disorders and to evaluate novel treatment strategies.
\end{abstract}

These authors contributed equally: Mario Van Poucke, Kimberley Stee, Sofie FM Bhatti, and Bart JG Broeckx

Supplementary information The online version of this article (https:// doi.org/10.1038/s41431-019-0432-3) contains supplementary material, which is available to authorized users.

Mario Van Poucke

Mario.VanPoucke@UGent.be

1 Department of Nutrition, Genetics and Ethology, Ghent University, Merelbeke, Belgium

2 Small Animal Department, Ghent University, Merelbeke, Belgium

3 Department of Pathology, Bacteriology and Poultry Diseases, Ghent University, Merelbeke, Belgium

\section{Introduction}

It has long been proven that research on human and canine diseases can benefit from each other because humans and dogs share hundreds of analogous diseases. On one hand, existing knowledge typically flows toward canine research, because human diseases are far more studied than canine diseases. On the other hand, new candidate genes for complex and/or rare human diseases are easily identified in dogs because they are often monogenic and common in dog

4 Department of Veterinary Medical Imaging and Small Animal Orthopaedics, Ghent University, Merelbeke, Belgium

5 Janssen Research \& Development, Janssen Pharmaceutica NV, Beerse, Belgium

6 Department of Pathology, Ghent University and Ghent University Hospital, Ghent, Belgium

7 Laboratory of Pharmaceutical Biotechnology, Ghent University, Ghent, Belgium 
breeds, and the availability of cells or tissues from a canine model might aid in the characterization of the underlying pathophysiology especially when appropriate human material is scarce [1].

This also applies to hereditary ataxias, a very heterogeneous group of neurological spinocerebellar disorders characterized by a lack of coordinated muscle movement. Ataxia can be present as an isolated symptom or as part of a syndrome. In $>100$ described human hereditary ataxias, similar phenotypes can be caused by variants in different genes and different variants in the same gene can cause different phenotypes [2]. Described genetic variants, often in genes from conserved pathways, are repeat expansions, SNVs and INDELs, and follow a dominant, recessive, Xlinked, or mitochondrial inheritance. The prevalence of hereditary ataxias in humans varies in different populations, but ranges between 1 and 9 in 100000 [3].

Ataxia-related phenotypes are also described in several dog breeds, with causal variants in KCNJ10, GRM1, ITPRI, SNX14, SPTBN2, CAPN1, ATP1B2, RAB24, and SEL1L (Supplementary Information File 1). Some of the KCNJ10 variants have been associated with a particular syndrome known as spinocerebellar ataxia, myokymia, seizures, or both (SAMS) in Jack Russel Terriers [4] and Malinois [5] dogs. Variants in the first five genes are also described to cause a spinocerebellar ataxia phenotype in humans, a variant in CAPNI has been reported to cause spastic paraplegia, but in the last three genes no human counterparts have been identified yet (Supplementary Information File 1).

Here, we report a new ataxia-related phenotype of slowly progressive spinocerebellar ataxia, paraparesis, and myokymic-like muscle contractions in a Malinois dog family caused by a truncating $S L C 12 A 6$ variant, and compared it with its human counterpart.

\section{Materials and methods}

\section{Clinical examination}

Four 6-12-month-old intact Malinois dogs (three males and one female) from two related litters were presented at the Small Animal Department of the Faculty of Veterinary Medicine of Ghent University for an uncoordinated gait since the age of 3-6 months. Two additional affected littermates were seen on video footage and blood was collected from 1 of them. A clinical and neurological examination was performed on all presented dogs. A complete blood count and serum biochemistry (including glucose and $\mathrm{Na}^{+}, \mathrm{K}^{+}, \mathrm{Cl}^{-}, \mathrm{Ca}^{2+}$, and $\mathrm{Mg}^{2+}$ electrolytes) was obtained from five dogs. Cerebrospinal fluid (CSF) analysis was performed in three dogs. Urinalysis (including electrolyte clearance for $\mathrm{Na}^{+}, \mathrm{K}^{+}, \mathrm{Cl}^{-}$, and $\mathrm{Ca}^{2+}$ ) was performed in two dogs. Electromyography (EMG) and motor nerve conduction velocity (MNCV) studies of the sciatic nerves were performed under general anesthesia in two dogs. EMG recordings were made from facial, truncal, and appendicular muscles of the front and hind limbs. Brain stem auditory evoked responses (BAER) were performed under sedation in one dog. A commercially available electrophysiological unit (Natus Synergy UltraPro, Acertys Healthcare NV, Aartselaar, Belgium) was used for electrodiagnostic recordings. Magnetic resonance imaging (MRI; 0.2 Tesla magnet) of the brain and complete spinal cord was done in one dog. CSF, electrophysiology, and MRI were performed on the day of euthanasia in all dogs. A summary of the clinical investigations performed in each of the six affected Malinois dogs (Fig. 1) is shown in Supplementary Information File 2.

\section{Pathological examination}

Post-mortem examination was performed in the four presented dogs, immediately after euthanasia. Both central and peripheral nervous tissue samples and skeletal muscle samples were collected, fixed in $10 \%$ neutral buffered formaldehyde, embedded in paraffin, sectioned at $5 \mu \mathrm{m}$, and stained with haematoxylin and eosin (H\&E). Additional histochemical stainings on selected sections included luxol fast blue (LFB) and toluidine blue (TB) on semi-thin sections. Immunohistochemistry (IHC) was performed for neurofilament (monoclonal mouse anti-human neurofilament protein clone 2F11, Cat No. M076229-2, Dako, Glostrup, Denmark), synaptophysin (monoclonal mouse anti-human synaptophysin clone DAK-SYNAP, Cat No. M731501-2, Dako, Glostrup, Denmark), glial fibrillary acidic protein (polyclonal rabbit anti-glial fibrillary acidic protein, Cat No. Z033401-2, Dako, Glostrup, Denmark) and ubiquitin (rabbit polyclonal ubiquitin IHC antibody, Cat No. IHC-00420, Bethyl laboratories, Montgomery TX, USA).

\section{Genetic analysis}

Ethylenediaminetetraacetic acid (EDTA) blood was sampled from five affected Malinois and 13 healthy family members (Fig. 1). In addition, EDTA blood was sampled from 118 reference Malinois dogs and 444 reference dogs from 17 breeds related to Malinois or known to suffer from ataxia (Supplementary Information File 3). DNA isolation from blood and the structural variant analysis of $K C N J 10$ was performed as described in Van Poucke et al. [5]. Details about the known ataxia-related causal variants in dogs are described in Supplementary Information File 1. Whole-exome sequencing was performed as described in Broeckx et al. [6]. The 


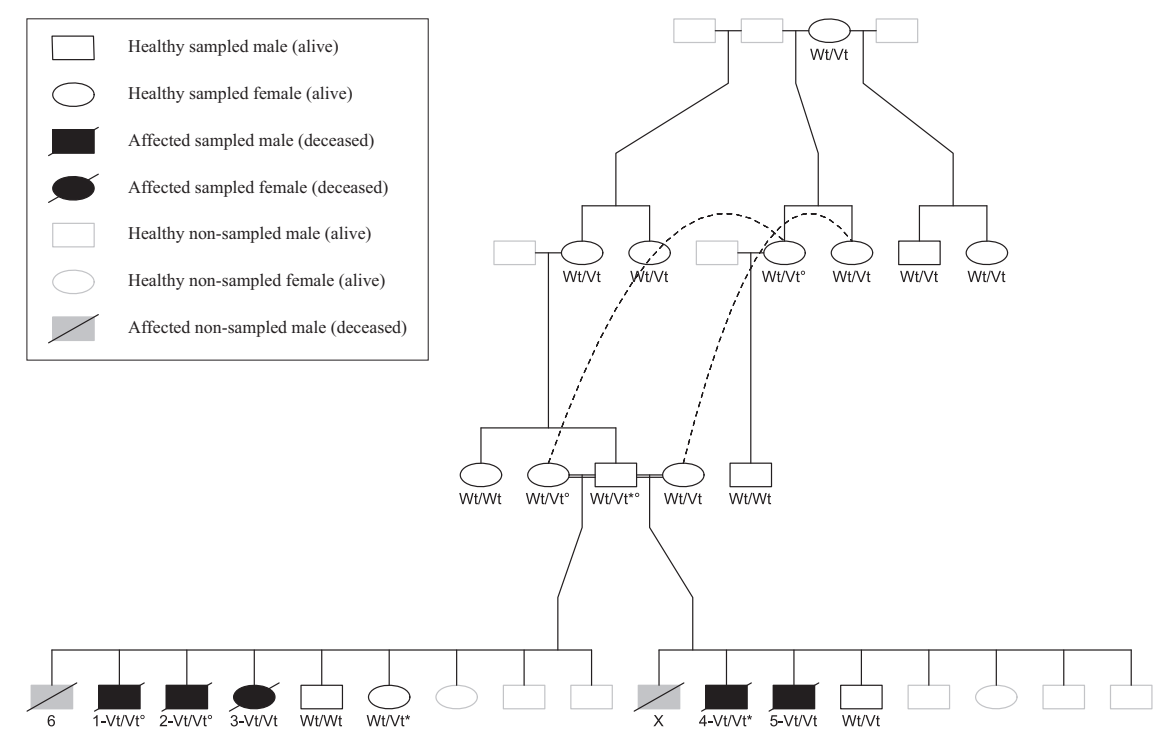

Fig. 1 Pedigree of the affected Malinois family (drawn with the kinship2 package in RStudio [35]). Numbers correspond to the investigated dogs described in Supplementary Information File 2. Dog X was killed because of the same symptoms but no video footage nor blood sample was available to confirm the diagnosis. Whole-exome

sequencing was performed on dogs marked with a degree sign. The SLC12A6 NC_006612.3(XM_014109414.2):c.178_181delinsCATCTCACTCAT genotype is shown as $\mathrm{Wt} / \mathrm{Wt}$, Wt/Vt, or $\mathrm{Vt} / \mathrm{Vt}$. Dogs with an asterisk carry 1 allele of the NC_006620.3: g.22141027_22141028insC variant [13]

reads were aligned to the reference genome using BWA v0.7.15 [7]. Duplicate reads were marked with Picard tools v2.1.1. Using the GATK v3.8-0, variants were called according to the GATK Best Practices [8]. From the total list of putative variants, only those were retained that (1) passed the "hard" quality filter suggested from the GATK Best Practices, (2) were not found in an internal variant database, nor in the public database of the online Variant Effect Predictor tool, (3) followed an autosomal recessive mode of inheritance, and (4) were predicted to be nonsynonymous $[9,10]$. Filtering was performed with VCFtools v0.1.14 and custom R-scripts [11]. A genotyping assay for the new variant is described in Supplementary Information File 4.

Cerebrum (unaffected), cerebellum (affected), and cervical spinal cord (affected) tissue samples were taken from two affected dogs. Tissue sampling, RNA isolation, and cDNA synthesis were performed as described by Van Poucke et al. [12]. Quantitative reverse transcription PCR (RT-qPCR) assays are described in Supplementary Information File 5.

\section{Results}

\section{Clinical features}

Clinical examination was unremarkable in all presented dogs, except for a mild palmigrade stance. Neurological examination revealed a severe generalized hypermetric ataxia (worse on the hindlimbs) associated with a mild to moderate degree of paraparesis and absent patellar reflexes in all dogs (Supplementary Information File 6a). Generalized (including tongue and eyelids) involuntary vermicular muscle contractions, strongly resembling myokymia but only triggered by sedation, were also seen in three dogs (Supplementary Information File 6b).

Complete blood count and serum biochemistry were normal. Urinalysis was unremarkable. EMG was silent in all clinically normal muscles. Unfortunately, EMG could not be performed in the muscles with involuntary vermicular contractions as those were short-lasting and transient. MNCV and BAER did not differ from age-matched Malinois control dogs. MRI of the brain and whole spinal cord and CSF analysis were unremarkable.

The ataxia, paraparesis, and palmigrade stance of all dogs progressively worsened, resulting in non-ambulatory paraparesis by 2.5-3 years of age (Supplementary Information File 6c) and therefore euthanasia was performed.

\section{Pathological features}

Weight of the four presented dogs ranged from 22 to $26 \mathrm{~kg}$ at the time of euthanasia (weight of healthy littermates at about the same age ranged between 28 and $35 \mathrm{~kg}$ ). At necropsy, no gross abnormalities were noted. The histopathologic findings were consistent with a severe bilateral symmetrical axonopathy of the white matter, characterized by prominent axonal swelling with vacuolation, affecting the whole spinal cord, as well as the dorsal and ventral nerve roots, brain stem, and cerebellum (in declining order of severity). Cerebrum, peripheral nerves, and skeletal 
muscles showed no or rare abnormalities. The lesions showed variation both in degree and in their location along the spinal cord, as well as within the same animal as in different animals, but the same pathways were consistently affected in all dogs. The most severe lesions were noted in some descending motor pathways (prominent in the ventral corticospinal tract and the vestibulospinal tract and to a lesser extent the lateral corticospinal tract) as well as in some sensory ascending pathways (prominent in the dorsal spinocerebellar tract and some mild lesions in the ventral spinocerebellar tract). The lesions consisted of sharply delineated swollen axons, often appearing optically empty ("axonal vacuoles") yet sometimes filled with a light eosinophilic, amorphous to slightly granular material ('axonal spheroids'). Axonal swelling was often extreme with diameters up to $140 \mu \mathrm{m}$. Neuronal perikarya in the cerebrum, cerebellum, brain stem, spinal cord, and dorsal root sensory ganglia appeared normal, except for the presence of some slight perikaryal retraction, probably representing an artifact. LFB stains and semi-thin sections stained with TB demonstrated well preserved myelin sheaths, both in the central and peripheral nervous system, surrounding the normal axons, the axonal spheroids and the empty vacuoles. Only around the extremely dilated axons, the myelin sheath appeared thinned or could not be visualized. IHC for neurofilament revealed a dilated aspect of almost all axons in the affected areas and scattered, extremely large axonal spheroids. The latter were also highlighted on IHC for synaptophysin. No abnormalities were detected on ubiquitin or glial fibrillary acidic protein IHC. See Supplementary Information File 7 for the histopathological lesions on $\mathrm{H} \& \mathrm{E}$, and on IHC for neurofilament and synaptophysin.

\section{Genetic analysis}

Because of the similarities of the syndrome in the dogs of the present study with SAMS and the fact that SAMS was so far only associated with $K C N J 10$ variants in dogs, we first tested the three previously described $K C N J 10$ variants in the affected Malinois dogs. The NC_006620.3 (XM_545752.6):c.627 C $>\mathrm{G}^{4}$ and c. $986 \mathrm{~T}>\mathrm{C}^{5}$ variants were not present. One dog carried one allele of the NC_006620.3:g.22141027_22141028insC [13] variant. Although this variant is not causal in heterozygous state, we analyzed this variant in the rest of the affected family and in 57 additional reference Malinois dogs, because it was the first time that this variant was detected in the Malinois breed. Also, the healthy father of the affected Malinois dog and a healthy offspring of that father carried one allele (Fig. 1), and the allele frequency in the reference Malinois dogs was $4.4 \%$. In addition, we did not find any of the eight other described ataxia-related canine variants in the affected Malinois dogs (Supplementary Information File 1). Next, we followed the candidate gene approach and performed a structural variant analysis on KCNJ1O in one affected Malinois dog. As no potential causal variants were found, whole-exome sequencing was performed on four animals (two healthy parents and two affected siblings; Fig. 1). A frameshift inducing INDEL in SLC12A6 was further investigated as the most likely causal variant after filtering.

SLC12A6 (solute carrier family 12 member 6, alias KCC3; Gene ID: 478239) is located on canine chromosome 30. Its canonical transcript is encoded in 25 exons and is translated into a 1151 aa long integral transmembrane protein involved in $\mathrm{K}^{+}-\mathrm{Cl}^{-}$cotransport, predicted to contain 12 transmembrane domains and large hydrophilic intracellular termini. Alternative transcripts, caused by alternative promoters, alternative transcript initiation sites, alternative exons (e.g., exon 1a and 1b) and alternative splicing (e.g., exon 2), give rise to a complex mix of isoforms in many tissue/cell types (exons are numbered like in Garneau et al.) [14-16].

The INDEL involves a 12-bp insertion (CATCTCACTCAT) and a 4-bp deletion (ATGA), most probably generated by a template-switch process with an inverted repeat and an inverted spacer (Fig. 2). The variant is located in exon 1a and causes a frameshift at codon 60 , leading to a premature stopcodon 14 codons downstream in all transcripts containing exon 1a (Fig. 2). The SLC12A6 NC_006612.3 (XM_014109414.2): c.178_181delinsCATCTCACTCAT (p. (Met60Hisfs*14)) variant was deposited in the EVA database (URL: https://www.ebi.ac.uk/eva/; Project: PRJEB30850; Analyses: ERZ802317).

From the 18 sampled Malinois family members, all five affected dogs were homozygous for the variant allele, 10 healthy dogs carried one variant allele and three healthy dogs did not carry the variant allele, following perfectly an autosomal recessive segregation (Fig. 1). The variant was not found in 118 additional reference Malinois dogs, neither in 444 reference dogs from 17 breeds related to Malinois or known to suffer from ataxia (Supplementary Information File 3). Because there were no false positives/negatives, odds ratios were infinite.

Because the variant does not affect transcripts containing exon 1b, RT-(q)PCR was performed, focusing on the first exons, to identify which SLC12A6 transcript variants (TVs) are transcribed in affected tissues (cerebellum and the cervical spinal cord) compared with unaffected tissue (cerebrum) from two affected Malinois dogs. Sequencing RT-PCR products identified TVs starting with both exon $1 \mathrm{a}$ or $1 \mathrm{~b}$, and both with or without exon 2 (data not shown). To quantify these endpoint detection results, we performed RT-qPCR with specific assays for the four observed TVs. Both TVs containing exon 1a (with or without exon 2) were highly transcribed, whereas both TVs containing exon $1 \mathrm{~b}$ were very weakly transcribed (at least 50-fold less) in all three investigated tissues from both animals (Supplementary Information File 5). 

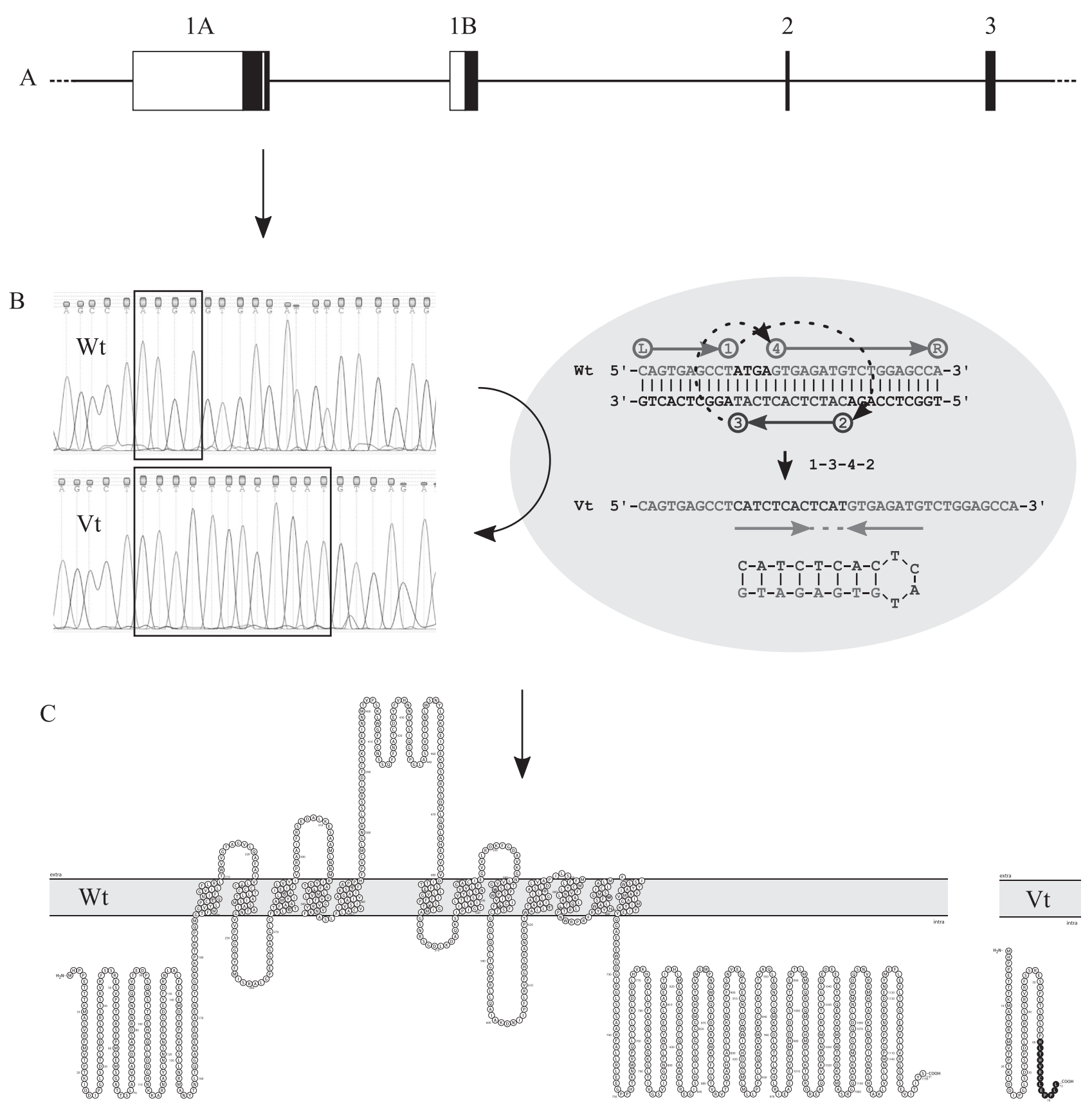

Fig. 2 Description, origin, location, and influence on protein structure of the SLC12A6 NC_006612.3(XM_014109414.2):c.178_181delinsCATCTCACTCAT(p.(Met60Hisfs*14)) variant. a shows a schematic representation of the genomic structure of the first exons of SLC12A6 (exons are numbered like in Garneau et al. [14]). White boxes represent exonic untranslated regions, black boxes represent exonic coding regions, and the white vertical bar represents the position of the

\section{Discussion}

SLC12A6 encodes one of the four distinct $\mathrm{K}^{+}-\mathrm{Cl}^{-}$cotransporters that belong to the cation- $\mathrm{Cl}^{-}$cotransporter family. Their structure, function, and regulation are highly conserved across evolution, and despite their high homology,
SLC12A6 variant. b shows the chromatograms of the wild type (Wt) and the variant type $(\mathrm{Vt})$, and the proposed origin of the INDEL by a template-switch process (1-3-4-2) with inverted repeat (arrows) and inverted spacer (dotted line) as described by Löytynoja and Goldman [36]. $\mathbf{c}$ shows the predicted structure of the canonical SLC12A6 protein $(\mathrm{Wt})$ and the truncated variant translated from the INDELcontaining transcript (Vt), drawn with Protter [37]

they exhibit unique patterns of distribution and fulfill distinct biophysical and physiological roles $[14,17]$. The functional properties of SLC12A6 are even more complex because it can exist in many isoforms that can be organized as homo- or hetero-oligomers with other cation- $\mathrm{Cl}^{-}$ cotransporters [18]. The SLC12A6 cotransporter is broadly 
expressed throughout the brain, spinal cord, and peripheral nervous system, among other various tissue locations $[14,19]$. It is inactive under isotonic conditions, but gets activated (by dephosphorylation of its $\mathrm{C}$-terminus) upon cell swelling where it regulates cell volume by the efflux of $\mathrm{K}^{+}$ and $\mathrm{Cl}^{-}$ions together with water molecules across the plasma membrane. It is therefore believed to have a key role in cell volume homeostasis and neuronal activity control $[14,17,18]$.

Naturally occurring disease-causing variants in SLC12A6 are so far only described in humans, where they cause "agenesis of the corpus callosum with peripheral neuropathy" (ACCPN, alias Andermann syndrome; phenotype MIM number 218000) [20]. It is a rare (prevalence rate of $<$ 1 in 1000000 individuals worldwide) [14], multisystemic disorder, characterized by sensorimotor polyneuropathy, variable degree of agenesis of the corpus callosum, mental retardation, and dysmorphic features [21, 22]. Psychotic episodes with visual and auditory hallucinations also have been reported [23]. The histopathologic lesions of ACCPN are a combination of axonal degeneration (axonal spheroids) with variable myelin swelling or loss (dependent on the location). They are most pronounced in the peripheral nervous system with progression to axonal loss and endoneurial and perineurial fibrosis. There is no obvious damage to neurons, no evidence of active myelin degradation or inflammation [22, 24, 25].

Most causal variants associated with ACCPN are randomly distributed truncating variants, owing to premature stop codons caused by INDEL, nonsense or splice site variants (recessive, homozygous, or compound heterozygous) $[14,17,18,20,26]$. Contrary to what would be expected, SLC12A6 mRNAs harboring premature termination codons are not degraded by nonsense-mediated mRNA decay, but are translated as truncated proteins [26]. They are associated to loss-of-function because of an aberrant structure or a defective transit to the plasma membrane [27]. Interestingly, Uyanik et al. [28] described a recessive missense variant (NG_007951.1(NM_133647.1):c.619 C > T (p. (Arg207Cys)), modifying a region crucial for oligomerization) associated with a milder form of ACCPN, and Kahle et al. [29] a dominant missense variant (NG_007951.1 (NM_133647.1):c.2971 A > G (p.(Thr991Ala)), abolishing a phosphorylation site crucial for deactivation) associated with a distinct form of ACCPN owing to a gain-of-function. Despite research on human patients with inherited diseasecausing variants or experiments in mouse, Xenopus, Caenorhabditis, and Drosophila model systems, the underlying pathological mechanisms that account for the neurological manifestations of ACCPN are still not clearly understood $[17,18,30]$.

Here, we describe the first non-human naturally occurring truncating SLC12A6 variant, segregating in a Malinois dog family descending from a common ancestor, owing to an INDEL in exon 1a, causing a frameshift at codon 60 and resulting in a premature stopcodon after 13 aberrant codons. It only affects TVs containing exon 1a and encodes the shortest truncated SLC12A6 protein reported so far, comprising only a part of the intracellular N-terminus (Fig. 2). As such, it can be considered as a loss-of-function variant. As in ACCPN patients, affected Malinois dog family members are homozygous for the truncating SLC12A6 variant, and heterozygotes are asymptomatic carriers. Because the SLC12A6 variant was only found in the affected Malinois family, it is likely a private variant because of a founder effect.

In agreement with what has been seen in humans [26], RT-qPCR results show that SLC12A6 mRNAs harboring a premature termination codon (i.e., TV1 and TV2) are not degraded by nonsense-mediated mRNA decay in dogs either, and will probably be translated into truncated proteins as well. The fact that the levels of transcripts containing exon $1 \mathrm{~b}$ (i.e., TV3 and TV4) are low (at least 50 times lower than transcripts containing exon 1a) and unchanged in affected tissues compared with unaffected, makes it very unlikely that they can take over the role of the predominant transcripts containing exon 1a. Unfortunately, there were no brain samples available from non-affected family members (including asymptomatic carriers) to compare their TV levels, nor isoform-specific antibodies to investigate SLC12A6 isoform expression via western blot analysis or IHC.

Electrophysiological findings associated with ACCPN in humans are abnormal resting activity on EMG, increased duration of motor unit potentials, increased polyphasia, decreased MNCV, and absent sensory action potentials $[21,22]$. Electrophysiology was entirely normal in the two investigated dogs, and furthermore no signs of sensorimotor neuropathy were found on histopathology. As EMG confirmation of myokymia could not be obtained in the muscles with involuntary vermicular contractions, we decided to refer to those as "myokymic-like muscle contractions".

Homozygous SLC12A6 global knockout in mice has been reported to reproduce the typical ACCPN sensorimotor neuropathy, as well as neurogenic hypertension, agerelated deafness, renal dysfunction, and a reduced threshold to develop epileptic seizures [31]. However, only minor changes of the corpus callosum have been reported in mice, and a complete agenesis has not yet been described in that species [17]. No signs of corpus callosum abnormalities nor signs of sensorineural deafness were seen in the investigated dogs. None of them developed epileptic seizures and their normal blood and urine analysis suggest normal renal function. Phenotypically, the current described syndrome seems quite similar to the SAMS syndrome previously reported in Malinois dogs [5] and Jack Russell Terriers 
[4, 32], both caused by a KCNJ1O variant. Still, some differences are undeniably present. The age of onset is younger in SAMS (6-8 weeks) and the progression to nonambulatory status is also more rapid in SAMS (before 6 months of age) compared with the dogs investigated here (respectively, 3-6 months and 2.5-3 years of age). Paraparesis and palmigrade stance were seen in this study but were not described in SAMS. Epileptic seizures have also been described in dogs with SAMS but were not seen here. Myokymia were clearly seen in dogs with SAMS when awake (confirmed by electrophysiological examination) and even progressed to neuromyotonia episodes in some cases, while the myokymic-like muscle contractions were only seen here when the dogs had been sedated and disappeared shortly after the induction of anesthesia. SAMS dogs also repetitively present some degree of subclinical sensorineural deafness, which was not seen here when a BAER test was performed [5, 32]. Those differences might be explained among others, by the fact that $K C N J 10$ encodes a voltagegated $\mathrm{K}^{+}$channel $[4,5]$, whereas SLC12A6 encodes an electroneutral $\mathrm{K}^{+}-\mathrm{Cl}^{-}$cotransporter in the brain and spinal cord.

The marked phenotypic differences between the human and canine phenotype of SLC12A6 variants are striking. Although speculative, it could be related to the fact that the human variants influence all TVs, in contrary to the canine one, where low levels of non-truncated (exon 1b-containing) TVs might induce another phenotype, or related to the length of the truncated protein that might have different functionalities. Quite pronounced phenotypic variations have already been reported between human patients, having distinct protein-truncating variants, as well as in mice $[14,17,18]$. Interestingly, as in humans where patients with ACCPN have extremely low body weights and heights [25], affected dogs weighted $\sim 25 \%$ less than age-matched littermates at the time of euthanasia. As our dogs did not display significant generalized muscle atrophy at time of euthanasia (only a moderate atrophy at the level of the pelvic limbs), and were generally smaller in size than their healthy siblings, we believe this decreased body weight to be mainly primary in origin.

The histopathologic findings show a strong correlation with the human ACCPN, as both display a severe axonopathy with striking unnoted neuronal damage, inflammation or gliosis. The dogs developed lesions in both central (mainly spinal cord and brain stem) and peripheral nervous system (mainly nerve roots), broadly consistent with the localization pattern in humans and mouse [25]. An important difference in dogs is the severe, bilateral symmetrical vacuolation of the spinal cord white matter, a feature not well described in humans. These minor interspecies differences can be explained by two complementary hypotheses. First, most human case reports are limited to biopsies of a peripheral sensory nerve (the sural nerve) and do not perform a complete necropsy [24, 28, 33, 34]. Second, dogs in this case report are all euthanized for humane reasons at an age of 1-3 years, in a disease state where they would not have spontaneously died. This is in contrast to the human patients with ACCPN who died a natural death at a moreadvanced stage of disease 20-30 years old [25]. As this is a chronic, progressive disease, an evolution in type of lesions and distribution pattern can be expected.

We conclude that the loss-of-function SLC12A6 NC_006612.3(XM_014109414.2): c.178_181delinsCATC TCACTCAT (p.(Met60Hisfs*14)) truncating variant causes an ataxia-related phenotype of slowly progressive spinocerebellar ataxia, paraparesis, and myokymic-like muscle contractions in Malinois dogs. Although the SLC12A6 variant in dogs resembles genetically the most frequently observed variants in humans, the clinical phenotype in dogs is quite different from ACCPN in humans. As this is the first report of a naturally occurring disease-causing $S L C 12 A 6$ variant in a non-human species, the canine model will be highly valuable to better understand the complex molecular pathophysiology of SLC12A6-related neurological disorders and to evaluate novel treatment strategies.

Acknowledgements We thank Sylvie Decraene, Sarah De Keulenaer, Ellen De Meester, Linda Impe, Caroline Rogiers, Dominique Vander Donckt, and Ruben Van Gansbeke for excellent technical assistance, Sameh A. Youssef and Martí Pumarola for their second opinion about the histological slides, as well as the breeders, owners, and veterinarians who collaborated to this study.

\section{Compliance with ethical standards}

Conflict of interest The authors declare that they have no conflict of interest.

Publisher's note: Springer Nature remains neutral with regard to jurisdictional claims in published maps and institutional affiliations.

\section{References}

1. Hytönen MK, Lohi H. Canine models of human rare disorders. Rare Dis. 2016;4:e1241362.3.

2. Wallace SE, Bird TD. Molecular genetic testing for hereditary ataxia: What every neurologist should know. Neurol Clin Pract. 2018:8:27-32.

3. Sandford E, Burmeister M. Genes and genetic testing in hereditary ataxias. Genes. 2014;5:586-603.

4. Gilliam D, O'Brien DP, Coates JR, Johnson GS, Johnson GC, Mhlanga-Mutangadura T, et al. A homozygous KCNJ10 mutation in Jack Russell Terriers and related breeds with spinocerebellar ataxia with myokymia, seizures, or both. J Vet Intern Med. 2014;28:871-7.

5. Van Poucke M, Stee K, Bhatti SF, Vanhaesebrouck A, Bosseler L, Peelman LJ, et al. The novel homozygous KCNJ10 c.986T>C (p. (Leu329Pro)) variant is pathogenic for the SeSAME/EAST homologue in Malinois dogs. Eur J Hum Genet. 2017;25:222-6.

6. Broeckx B, Hitte C, Coopman F, Verhoeven GE, De Keulenaer S, De Meester E, et al. Improved canine exome designs, featuring 
ncRNAs and increased coverage of protein coding genes. Sci Rep. 2015;5:12810.

7. Li H, Durbin R. Fast and accurate long-read alignment with Burrows-Wheeler transform. Bioinformatics. 2010;26:589-95.

8. Van der Auwera GA, Carneiro MO, Hartl C, Poplin R, Del Angel G, Levy-Moonshine A, et al. From FastQ data to high-confidence variant calls: the genome analysis toolkit best practices pipeline. Curr Protoc Bioinformatics. 2013;43:11.10.1-11.10.33.

9. McLaren W, Gil L, Hunt SE, Riat HS, Ritchie GR, Thormann A, et al. The Ensembl variant effect predictor. Genome Biol. 2016;17:122.

10. Broeckx B, Peelman L, Saunders J, Deforce D, Clement L. Using variant databases for variant prioritization and to detect erroneous genotype-phenotype associations. BMC Bioinformatics. 2017;18: 535.

11. Danecek P, Auton A, Abecasis G, Albers CA, Banks E, DePristo MA, et al. The variant call format and VCFtools. Bioinformatics. 2011;27:2156-8.

12. Van Poucke M, Martlé V, Van Brantegem L, Ducatelle R, Van Ham L, Bhatti S, et al. A canine orthologue of the human GFAP c.716G4A (p.Arg239His) variant causes Alexander disease in a Labrador retriever. Eur J Hum Genet. 2016;24:852-6.

13. Gast AC, Metzger J, Tipold A, Distl O. Genome-wide association study for hereditary ataxia in the Parson Russell Terrier and DNAtesting for ataxia-associated mutations in the Parson and Jack Russell Terrier. BMC Vet Res. 2016;12:225.

14. Garneau AP, Marcoux AA, Frenette-Cotton R, Mac-Way F, Lavoie JL, Isenring P. Molecular insights into the normal operation, regulation, and multisystemic roles of $\mathrm{K}+-\mathrm{Cl}-$ cotransporter 3 (KCC3). Am J Physiol Cell Physiol. 2017;313: C516-C532.

15. Hiki K, D'Andrea RJ, Furze J, Crawford J, Woollatt E, Sutherland GR, et al. Cloning, characterization, and chromosomal location of a novel human K+-Cl- cotransporter. J Biol Chem. 1999;274: 10661-7.

16. Mount DB, Mercado A, Song L, Xu J, George AL, Delpire E, et al. Cloning and characterization of $\mathrm{KCC} 3$ and $\mathrm{KCC} 4$, new members of the cation-chloride cotransporter gene family. J Biol Chem. 1999;274:16355-62.

17. Flores B, Schornack CC, Delpire E. A role for KCC3 in maintaining cell volume of peripheral nerve fibers. Neurochem Int. 2019;123:114-24.

18. Kahle KT, Khanna AR, Alper SL, Adragna NC, Lauf PK, Sun D, et al. $\mathrm{K}-\mathrm{Cl}$ cotransporters, cell volume homeostasis, and neurological disease. Trends Mol Med. 2015;21:513-23.

19. Bowerman M, Salsac C, Bernard V, Soulard C, Dionne A, Coque E, et al. KCC3 loss-of-function contributes to Andermann syndrome by inducing activity-dependent neuromuscular junction defects. Neurobiol Dis. 2017;106:35-48.

20. Howard HC, Mount DB, Rochefort D, Byun N, Dupré N, Lu J, et al. The $\mathrm{K}-\mathrm{Cl}$ cotransporter $\mathrm{KCC} 3$ is mutant in a severe peripheral neuropathy associated with agenesis of the corpus callosum. Nat Genet. 2002;32:384-92.

21. Larbrisseau A, Vanasse M, Brochu P, Jasmin G. The Andermann syndrome: agenesis of the corpus callosum associated with mental retardation and progressive sensorimotor neuronopathy. Can J Neurol Sci. 1984;11:257-61.

22. Hauser BE, Bittner R, Liegl C, Bernert G, Zeitlhofer J. Occurrence of Andermann syndrome out of French Canada - agenesis of the corpus callosum with neuronopathy. Neuropediatrics. 1993;24:107-10.

23. Filteau MJ, Pourcher E, Bouchard RH, Baruch P, Mathieu J, Bédard F, et al. Corpus callosum agenesis and psychosis in andermann syndrome. Arch Neurol. 1991;48:1275-80.

24. Deleu D, Bamanikar SA, Muirhead D, Louon A. Familial progressive sensorimotor neuropathy with agenesis of the corpus callosum (Andermann Syndrome): a clinical, neuroradiological and histopathological study. Eur Neurol. 1997;37:104-9.

25. Auer R, Laganière J, Robitaille Y, Richardson J, Dion PA, Rouleau GA, et al. KCC3 axonopathy: neuropathological features in the central and peripheral nervous system. Mod Pathol. 2016;29:962-76

26. Salin-Cantegrel A, Shekarabi M, Holbert S, Dion P, Rochefort D, Laganière $\mathrm{J}$, et al. HMSN/ACC truncation mutations disrupt braintype creatine kinase-dependant activation of $\mathrm{K}+/ \mathrm{Cl}-\mathrm{co}-$ transporter 3. Hum Mol Genet. 2008;17:2703-11.

27. Salin-Cantegrel A, Rivière JB, Shekarabi M, Rasheed S, Dacal S, Laganière $\mathrm{J}$, et al. Transit defect of potassium-chloride Co-transporter 3 is a major pathogenic mechanism in hereditary motor and sensory neuropathy with agenesis of the corpus callosum. J Biol Chem. 2011;286:28456-65.

28. Uyanik G, Elcioglu N, Penzien J, Gross C, Yilmaz Y, Olmez A, et al. Novel truncating and missense mutations of the KCC3 gene associated with Andermann syndrome. Neurology. 2006;66:1044-8.

29. Kahle KT, Flores B, Bharucha-Goebel D, Zhang J, Donkervoort $\mathrm{S}$, Hegde M, et al. Peripheral motor neuropathy is associated with defective kinase regulation of the KCC3 cotransporter. Sci Signal. 2016;9:ra77.

30. Salin-Cantegrel A, Shekarabi M, Rasheed S, Charron FM, Laganière $\mathrm{J}$, Gaudet $\mathrm{R}$, et al. Potassium-chloride cotransporter 3 interacts with Vav2 to synchronize the cell volume decrease response with cell protrusion dynamics. PLoS ONE. 2013;8: e65294.

31. Ding J, Delpire E. Deletion of KCC3 in parvalbumin neurons leads to locomotor deficit in a conditional mouse model of peripheral neuropathy associated with agenesis of the corpus callosum. Behav Brain Res. 2014;274:128-36.

32. Bhatti SF, Vanhaesebrouck A, Van Soens I, Martlé VA, Polis IE, Rusbridge C, et al. Myokymia and neuromyotonia in 37 Jack Russell terriers. Vet J. 2011;189:284-8.

33. Rudnik-Schöneborn $\mathrm{S}$, Hehr $\mathrm{U}$, von Kalle $\mathrm{T}$, Bornemann A, Winkler J, Zerres K. Andermann Syndrome can be a phenocopy of hereditary motor and sensory neuropathy - report of a discordant sibship with a compound heterozygous mutation of the KCC3 gene. Neuropediatrics. 2009;40:129-33.

34. Lourenço CM, Dupré N, Rivière JB, Rouleau GA, Marques VD, Genari AB, et al. Expanding the differential diagnosis of inherited neuropathies with non-uniform conduction: Andermann syndrome. J Peripher Nerv Syst. 2012;17:123-7.

35. Sinnwell JP, Therneau TM, Schaid DJ. The kinship2 R package for pedigree data. Hum Hered. 2014;78:91-93.

36. Löytynoja A, Goldman N. Short template switch events explain mutation clusters in the human genome. Genome Res. 2017;27: 1039-49.

37. Omasits U, Ahrens CH, Müller S, Wollscheid B. Protter: interactive protein feature visualization and integration with experimental proteomic data. Bioinformatics. 2014;30:884-6. 